guiding antibiotic prescribing. Our service evaluation showed that despite this, introducing it into a hospital setting is challenging. If the algorithm had been followed correctly $65 \%$ of patients would not have received antibiotics. Physicians often rely on clinical judgement and well-known markers of infections such as CRP and WCC. Education of physicians along with timely availability of the test play significant roles. We intend to persevere and report our results aiming to improve the use of PCT within our hospital.

\section{P114 C-REACTIVE PROTEIN AT ONSET PREDICTS SYMPTOM RESOLUTION IN ACUTE EXACERBATIONS OF COPD}

doi:10.1136/thx.2010.150987.15

A J Mackay, G C Donaldson, A R C Patel, J R Hurst, J A Wedzicha. Academic Unit of Respiratory Medicine, UCL Medical School, Royal Free Campus, London, UK

Introduction Patients with chronic obstructive pulmonary disease (COPD) may not recover swiftly from exacerbations. In $23 \%$ of patients, symptoms do not recover within 35 days. ${ }^{1}$ It would be valuable for clinicians to have a systemic biomarker that taken at initial consultation for an exacerbation could predict exacerbation length. We hypothesised that inflammation, measured by serum Creactive protein (CRP) predicts the time to symptomatic resolution. Methods We analysed daily diary cards prospectively collected from the London COPD cohort between October 1995 and January 2010. All patients had an $\mathrm{FEV}_{1} \leq 80 \%$ predicted and an $\mathrm{FEV}_{1} / \mathrm{FVC}$ ratio $\leq 70 \%$. An exacerbation was defined as an increase for 2 consecutive days in respiratory symptoms, with at least one major symptom (dyspnoea, sputum purulence or volume) plus another major or minor symptom (wheeze, cold sore throat and cough). Exacerbations were separated by five symptom free days. Patients were assessed and sampled at clinic visits within a median of 2 days of exacerbation onset. Exacerbation length was defined as the number of days from onset that increased airway symptoms were still recorded, and resolution time was defined as the time from clinic assessment to symptom cessation.

Results 268 patients with $\geq 365$ days of diary card data experienced at least one exacerbation. Median baseline CRP was $4 \mathrm{mg} / \mathrm{l}$. CRP at onset was available for 172 recovered exacerbations (Abstract P114 Figure 1). Mean age was 68.0 years (SD 8.3), with mean $\mathrm{FEV}_{1} 1.111$ (0.45), $\mathrm{FEV}_{1}$ predicted $44.6 \%$ (16.2). Patients with a CRP $<4 \mathrm{mg} / \mathrm{l}$ at initial clinic review had a significantly shorter resolution time than patients with $\mathrm{CRP} \geq 4 \mathrm{mg} / \mathrm{l}$, median 6 (IOR 3-11; $\mathrm{n}=46$ ) vs 10 $(5-23 ; \mathrm{n}=126)$ days, $\mathrm{p}=0.0017$. Cox proportional hazard risk models with adjustment for repeated measures showed that CRP predicted both exacerbation length $(\mathrm{p}=0.039)$ and resolution time $(\mathrm{p}=0.029)$.

Conclusions COPD exacerbations can occur without an elevated systemic inflammatory response. These events are associated with a

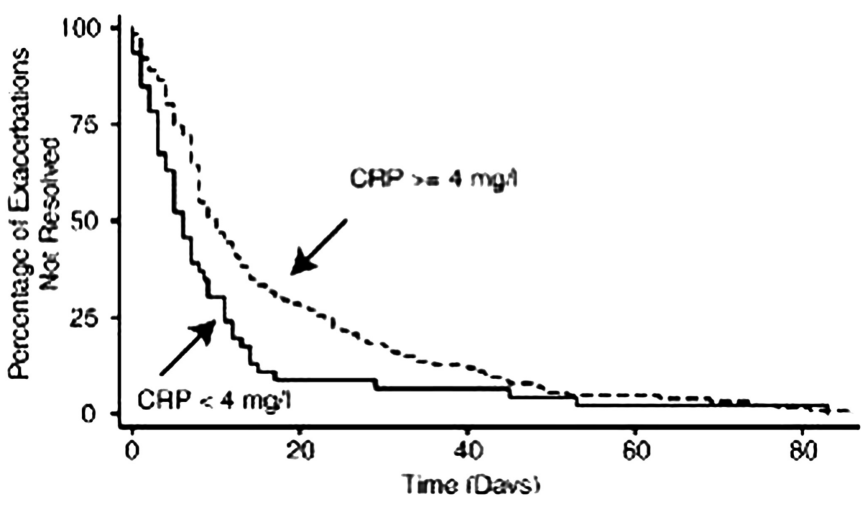

Abstract P114 Figure 1 Kaplan-Meier plot of symptom resolution by high and low CRP at exacerbation onset. short resolution time. Thus, using CRP as a biomarker, it may be possible to identify patients that have a short recovery period and potentially may not require systemic treatment for their exacerbation.

\section{REFERENCE}

1. Perera, et al. Inflammatory changes, recovery and recurrence at COPD exacerbation ERJ 2007;29:527-34.

\section{P115 VITAMIN D BINDING PROTEIN IN COPD EXACERBATIONS}

\author{
doi:10.1136/thx.2010.150987.16
}

${ }^{1} \mathrm{M}$ Newnham, ${ }^{2} \mathrm{R}$ Carter, ${ }^{2} \mathrm{~L}$ Sapey, ${ }^{3} \mathrm{R}$ A Stockley, ${ }^{2} \mathrm{~A} M$ Wood. ${ }^{1}$ Heart of England NHS Trust, Birmingham, UK; ${ }^{2}$ University of Birmingham, Birmingham, UK; ${ }^{3}$ University Hospitals Birmingham, Birmingham, UK

Introduction Vitamin D binding protein (DBP) plays a role in macrophage activation and neutrophil chemotaxis, both functions being relevant to COPD and airway infection. We have shown previously that $\mathrm{DBP}$ is present in the COPD lung and relates directly to macrophage activation. ${ }^{1}$ Transcription of DBP is stimulated by inflammatory cytokines, ${ }^{2}$ which tend to be raised in exacerbations. $\mathrm{DBP}$ is also cleaved from its binding site on neutrophils by the action of neutrophil elastase. ${ }^{3}$ We reasoned that DBP would be elevated in the lung and systemic circulation during exacerbations of COPD compared to the stable state.

Methods 20 patients with a known diagnosis of COPD were studied in the stable state and at the start of an exacerbation. Circulating CRP, elastase, AAT and DBP were measured at each time point, together with sol phase DBP, AAT and CRP, where sufficient sample was available $(n=9)$. All clinical parameters were studied in the stable state only. Relationships between exacerbation and stable state were sought using pair-wise tests for each individual, whilst group relationships between DBP, CRP and elastase were sought using Spearman's rank correlation.

Results No significant differences between stable state and exacerbation were seen for circulating or sol phase DBP (both $\mathrm{p}>0.4$ ). Circulating CRP and AAT were significantly higher in exacerbation (mean difference $3.95, \mathrm{p}<0.0001$ and $137.39, \mathrm{p}=0.03$ respectively), as were sol phase CRP and AAT (mean difference 18.29, $p=0.003$ and $0.60, p=0.009$ respectively). DBP did not relate to elastase in either stable state $(p=0.61)$ or exacerbation $(p=0.17)$, nor to CRP $(p=0.71$ and 0.98 , respectively).

Conclusions The level of local and systemic inflammation, with consequent elastase release, during a COPD exacerbation is insufficient to alter DBP levels. As such it is unlikely that DBP-derived macrophage activation is important in their pathogenesis or resolution.

\section{REFERENCES}

1. Wood, et al. Thorax 2009;54(Suppl IV):A3

2. Guha, et al. Hepatology 1995;21:1675-81.

3. DiMartino, et al. J Immunol 2001;166:2688-94.

P116 SERUM PARC (CCL18) AND EXACERBATION FREQUENCY IN COPD

doi:10.1136/thx.2010.150987.17

C G George, J K Quint, R J Sapsford, G C Donaldson, J A Wedzicha, J R Hurst. University College London, London, UK

Introduction Patients with Chronic Obstructive Pulmonary Disease (COPD) experience intermittent exacerbations. The frequency of exacerbations varies amongst patients but the "Frequent Exacerbator' appears to be an independent disease phenotype. As frequent exacerbations are associated with more rapid lung function decline, a validated biomarker is needed to identify those susceptible, prompting rigorous medical treatment and aiding selective 
recruitment to clinical trials. Pulmonary and Activation-Regulated Chemokine (PARC/CCL18) is a plausible biomarker based on previous reports in other respiratory diseases, making it worthy of study in COPD.

Methods PARC was measured using ELISA in serum samples from 115 patients enrolled in The London COPD cohort, including 44 paired samples taken at baseline and exacerbation (pre-treatment). PARC was assessed with relation to exacerbation frequency and other inflammatory markers.

Results The study cohort comprised of 77 males, 34 current smokers, mean age 69.6 years (SD 9.1), $\mathrm{FEV}_{1} 1.13$ (0.47) 1 (45.3 (18.0)\% predicted), baseline PARC concentration $124 \mathrm{ng} / \mathrm{ml}$ (40.4), median (IOR) exacerbation frequency 1.8/year (0.6-3.0). Higher PARC concentration was associated with more frequent exacerbations $(\mathrm{r}=0.22, \mathrm{p}=0.035)$. PARC was not related to age, sex, BMI, disease severity $\left(\mathrm{FEV}_{1}\right)$, or smoking pack years (all $\mathrm{p}>0.05$ ). Significantly lower PARC concentrations were found in current smokers compared to ex-smokers, $112 \mathrm{ng} / \mathrm{ml}$ vs $130 \mathrm{ng} / \mathrm{ml}$ respectively ( $\mathrm{p}=0.036$ ). PARC did not change from baseline to exacerbation $(131 \mathrm{ng} / \mathrm{ml} \mathrm{vs}$ $125 \mathrm{ng} / \mathrm{ml}, \mathrm{p}=0.256$ ), and the correlation between PARC in the two states was highly significant $(\mathrm{r}=0.53, \mathrm{p}<0.0001)$. PARC was related to baseline CRP $(r=0.28, p=0.013)$ and blood eosinophil count $(r=0.39, p=0.001)$, with no significant associations at exacerbation, and no relationship with neutrophils or total white blood cell count.

Conclusion A relationship has been demonstrated between serum PARC concentration and exacerbation frequency in patients with COPD. Correlations between PARC, eosinophils and CRP indicate that this biomarker may identify a subset of patients with a particular inflammatory profile, suggesting specific treatment options. Further work should be carried out to explore the relevance of PARC as a biomarker in the COPD population.

\section{P117 COMPARISON OF INDICES OF NUTRITIONAL STATUS IN PREDICTION OF IN-HOSPITAL MORTALITY AND EARLY READMISSION OF PATIENTS WITH ACUTE EXACERBATIONS OF COPD}

doi:10.1136/thx.2010.150987.18

1J Steer, ${ }^{1} E$ Norman, ${ }^{2} \mathrm{G}$ J Gibson, ${ }^{1} \mathrm{~S}$ C Bourke. ${ }^{1}$ North Tyneside General Hospital, North Shields, Tyne and Wear, UK; ${ }^{2}$ Institute of Cellular Medicine, Newcastle University, Newcastle-upon-Tyne, UK

Introduction and objectives In patients hospitalised with an acute exacerbation of COPD (AECOPD), low body mass index (BMI) predicts in-hospital death. The Malnutrition Universal Screening Tool (MUST) incorporates BMI and patient-reported weight loss over the previous 6 months to provide an overall assessment of malnutrition risk. It predicts mortality in elderly hospitalised patients ${ }^{1}$ but, to our knowledge, the prognostic value of this tool in AECOPD has not been previously reported.

Methods We prospectively identified patients hospitalised with AECOPD. We investigated the ability of BMI, self-reported weight loss and MUST score to predict in-hospital mortality and 28-day readmission. BMI $<18.5 \mathrm{kgm}^{-2}$ was considered underweight (World Health Organisation, 2004). Odds Ratios (OR) were calculated using normal BMI, weight loss $<5 \%$, and MUST score 0 as reference values. Results 608 patients were included; mean (SD) age 72.8 (10.2) years, $55.8 \%$ female, mean (SD) $\mathrm{FEV}_{1}$ (if performed within 2 years of admission, $n=398) 43.5$ (18) \% predicted. 61 (10\%) patients died inhospital $(6.9 \%$ in those with simple exacerbations, $16.5 \%$ in exacerbations associated with pneumonia). Of patients surviving to discharge, 95 (17.4\%) were readmitted within 28 days (Abstract P117 Table 1). In-hospital mortality was predicted by BMI $<18.5 \mathrm{kgm}^{-2}$ (OR 2.5, 95\% CI 1.27 to $4.91, \mathrm{p}=0.008$ ) whereas weight loss $>10 \%$ predicted early readmission (OR 3.90, 95\% CI 2.09 to $7.28, \mathrm{p}<0.001$ ). A high risk of malnutrition (MUST $\geq 2$ ) was the only measurement that significantly predicted both inhospital mortality (OR 2.10, 95\% CI 1.18 to 3.74, $\mathrm{p}=0.011$ ) and early readmission to hospital (OR 1.71, 95\% CI 1.04 to 2.83, $\mathrm{p}=0.034)$. BMI within the overweight range appeared to be protective against early readmission (OR $0.54,95 \%$ CI 0.29 to 0.99 , $\mathrm{p}=0.046$ ).

Conclusion In patients hospitalised with AECOPD, indices of nutritional status are important predictors of outcome. Of interest, BMI and self-reported weight loss predict different outcome measures (in-hospital death and early readmission respectively). A high risk of malnutrition (MUST score $\geq 2$ ) is potentially a useful predictor of both in-hospital mortality and early hospital readmission and we suggest that it should be assessed routinely.

Abstract P117 Table 1 Nutritional measurements and their relationship to outcome

\begin{tabular}{|c|c|c|c|c|c|c|c|c|c|c|}
\hline & BMI (k & $\left.\operatorname{sm}^{-2}\right)$ & & & Weig & ght loss & & MUS & & \\
\hline & $<18.5$ & $18.5-24.9$ & $25-29.9$ & $>30$ & $<5$ & $5-10$ & $>10$ & 0 & 1 & $\geq 2$ \\
\hline$\%$ & 17.8 & 37.5 & 25 & 19.7 & 79.3 & 11.2 & 9.5 & 64.8 & 10.4 & 24.8 \\
\hline $\begin{array}{l}\text { OR for } \\
\text { in-hospital } \\
\text { mortality }\end{array}$ & $2.50^{*}$ & 1 & 1.03 & 0.89 & 1 & 1.52 & 1.59 & 1 & 1.46 & $2.10^{*}$ \\
\hline $\begin{array}{l}\text { OR for early } \\
\text { readmission }\end{array}$ & 0.86 & 1 & $0.54^{*}$ & 0.80 & 1 & 1.36 & $3.90^{*}$ & 1 & 1.24 & $1.71^{*}$ \\
\hline
\end{tabular}

${ }^{*}$ Significance $<0.05$.

\section{REFERENCE}

1. Henderson S, Moore N, Lee E, Witham MD. Do the malnutrition universal screening tool (MUST) and Birmingham nutrition risk (BNR) score predict mortality in older hospitalised patients? BMC Geriatrics 2008;8

\section{P118 PREDICTING SURVIVAL IN PATIENTS ADMITTED TO HOSPITAL WITH AN ACUTE EXACERBATION OF CHRONIC OBSTRUCTIVE PULMONARY DISEASE (COPD)}

doi:10.1136/thx.2010.150987.19

K Williamson, A Sullivan, N Folland, S Rees, S Gompertz. Queen Elizabeth Hospital, Birmingham, UK

Introduction COPD is the fifth biggest cause of death in the UK. It can be difficult to predict when these patients are coming to the end of their life. There are many clinical tools available to aid prediction of death. ${ }^{12}$ As part of a wider COPD audit we assessed whether one of these could identify which patients admitted with an exacerbation should be referred for specialist palliative care.

Method 50 consecutive patients admitted to an acute hospital with exacerbations of COPD were assessed between August and September 2009. Gold Standards Framework ${ }^{1}$ indicators for organ disease and other general predictors of end-stage illness were recorded. Where available (33/50), we calculated a modified BODE score (without the measure of exercise capacity). At 10 months, we assessed how many patients had survived.

Results $30 \%(15 / 50)$ of the patients had died by 10 months. Median BOD score at admission was five in survivors and in those who had died. The remaining data are summarised in Abstract P118 Table 1. Discussion In our group of patients a modified BODE score was not helpful in predicting outcome at 10 months. Recurrent admissions, oxygen therapy, severe breathlessness/dependence and resistant organisms were more prevalent in the group that died. These data 